\title{
Applications of Fractional Calculus to Newtonian Mechanics
}

\author{
Gabriele U. Varieschi \\ Department of Physics, Loyola Marymount University, Los Angeles, CA, USA \\ Email: gvarieschi@lmu.edu
}

How to cite this paper: Varieschi, G.U. (2018) Applications of Fractional Calculus to Newtonian Mechanics. Journal of Applied Mathematics and Physics, 6, 1247-1257. https://doi.org/10.4236/jamp.2018.66105

Received: April 6, 2018

Accepted: June 18, 2018

Published: June 21, 2018

Copyright $\odot 2018$ by author and Scientific Research Publishing Inc. This work is licensed under the Creative Commons Attribution International License (CC BY 4.0).

http://creativecommons.org/licenses/by/4.0/

\begin{abstract}
We investigate some basic applications of Fractional Calculus (FC) to Newtonian mechanics. After a brief review of FC, we consider a possible generalization of Newton's second law of motion and apply it to the case of a body subject to a constant force. In our second application of FC to Newtonian gravity, we consider a generalized fractional gravitational potential and derive the related circular orbital velocities. This analysis might be used as a tool to model galactic rotation curves, in view of the dark matter problem. Both applications have a pedagogical value in connecting fractional calculus to standard mechanics and can be used as a starting point for a more advanced treatment of fractional mechanics.
\end{abstract}

\section{Keywords}

Fractional Calculus, Fractional Differential Equations, Fractional Mechanics

\section{Introduction}

Fractional Calculus (FC) is a natural generalization of calculus that studies the possibility of computing derivatives and integrals of any real (or complex) order [1] [2] [3], i.e., not just of standard integer orders, such as first-derivative, second-derivative, etc.

The history of FC started in 1695 when l'Hôpital raised the question as to the meaning of taking a fractional derivative such as $\mathrm{d}^{1 / 2} y / \mathrm{d} x^{1 / 2}$ and Leibniz replied [2]: “...This is an apparent paradox from which, one day, useful consequences will be drawn."

Since then, eminent mathematicians such as Fourier, Abel, Liouville, Riemann, Weyl, Riesz, and many others contributed to the field, but until lately FC has played a negligible role in physics. However, in recent years, applications of FC 
to physics have become more common [4] [5] in fields ranging from classical and quantum mechanics, nuclear physics, hadron spectroscopy, and up to quantum field theory.

In theoretical physics we can now study the fractional equivalent of many standard physics equations [4]: frictional forces, harmonic oscillator, wave equations, Schrödinger and Dirac equations, and several others. In applied physics [5], FC methods can be used in the description of chaotic systems and random walk problems, in polymer material science, in biophysics, and other fields.

In this paper, we will review elementary definitions and methods of fractional calculus and fractional differential equations. We will then apply these concepts to some basic problems in Newtonian mechanics, such as possible generalizations of Newton's second law of motion and applications of FC to Newtonian gravity.

\section{Fractional Calculus: A Brief Review}

Unlike standard calculus, there is no unique definition of derivation and integration in FC. Historically, several different definitions were introduced and used (for complete details see, for example, Refs. [1] and [2]). All proposed definitions reduce to standard derivatives and integrals for integer orders $n$, but they might not be fully equivalent for non-integer orders of differ-integration ${ }^{1}$.

To gain an intuitive perspective of fractional derivatives [4], we consider some elementary functions such as the exponential function $\mathrm{e}^{k x}$, trigonometric functions $\sin (k x)$ or $\cos (k x)$, and simple powers $x^{k}$, where $k$ is some constant. It is easy to obtain recursive relations for derivatives of integer order $n$ :

$$
\begin{gathered}
\frac{\mathrm{d}^{n} \mathrm{e}^{k x}}{\mathrm{~d} x^{n}}=k^{n} \mathrm{e}^{k x} \\
\frac{\mathrm{d}^{n} \sin (k x)}{\mathrm{d} x^{n}}=k^{n} \sin \left(k x+\frac{\pi}{2} n\right) \\
\frac{\mathrm{d}^{n} x^{k}}{\mathrm{~d} x^{n}}=\frac{k !}{(k-n) !} x^{k-n}
\end{gathered}
$$

These relations can be easily generalized to real or imaginary order $q$, with appropriate gamma functions replacing the factorials when necessary:

$$
\begin{gathered}
\frac{\mathrm{d}^{q} \mathrm{e}^{k x}}{\mathrm{~d} x^{q}}=k^{q} \mathrm{e}^{k x} ; k \geq 0 \\
\frac{\mathrm{d}^{q} \sin (k x)}{\mathrm{d} x^{q}}=k^{q} \sin \left(k x+\frac{\pi}{2} q\right) ; k \geq 0
\end{gathered}
$$

\footnotetext{
${ }^{1}$ In FC derivation and integration are often treated and defined as a single operation-with the order $q$ respectively taken as a positive or negative real number-hence the names differ integrals, differ integration, etc. Also, the name fractional calculus is actually a misnomer, since the order of differ integration can be any real (or complex) number. A better name for this field might be "Differ integration to an arbitrary order," or similar.
} 


$$
\frac{\mathrm{d}^{q} x^{k}}{\mathrm{~d} x^{q}}=\frac{\Gamma(k+1)}{\Gamma(k-q+1)} x^{k-q} ; x \geq 0, k \neq-1,-2, \cdots
$$

with the first two functions restricted to $k \geq 0$ and the third one restricted to $x \geq 0$, to ensure the uniqueness of the above definitions.

These three approaches to fractional derivation were introduced respectively by Liouville, Fourier, and Riemann and led to immediate generalizations for analytic functions expanded in series of exponential, trigonometric, or power functions. For example, the fractional derivative of a function $f(x)$, according to Liouville, can be defined as

$$
\begin{gathered}
f(x)=\sum_{k=0}^{\infty} a_{k} \mathrm{e}^{k x} \\
\frac{\mathrm{d}^{q} f(x)}{\mathrm{d} x^{q}}=\sum_{k=0}^{\infty} a_{k} k^{q} \mathrm{e}^{k x} .
\end{gathered}
$$

Applying instead the Riemann definition of fractional derivatives for power functions to the case of a constant $C$, we obtain:

$$
\frac{\mathrm{d}^{q} C}{\mathrm{~d} x^{q}}=\frac{\mathrm{d}^{q}\left(C x^{0}\right)}{\mathrm{d} x^{q}}=\frac{C x^{-q}}{\Gamma(1-q)},
$$

i.e., the derivative of a constant is not equal to zero in FC, unless this condition is assumed as an additional postulate as in the so-called Caputo derivative [4].

More general definitions of fractional differ integrals exist in the literature, such as the Grünwald formula [1]:

$$
\frac{\mathrm{d}^{q} f}{[\mathrm{~d}(x-a)]^{q}}=\lim _{N \rightarrow \infty}\left\{\frac{\left[\frac{x-a}{N}\right]^{-q}}{\Gamma(-q)} \sum_{j=0}^{N-1} \frac{\Gamma(j-q)}{\Gamma(j+1)} f\left(x-j\left[\frac{x-a}{N}\right]\right)\right\},
$$

which involves only evaluations of the function itself and can be used for both positive and negative values of $q$. Another general definition is the Riemann-Liouville fractional integral [1]:

$$
\frac{\mathrm{d}^{q} f}{[\mathrm{~d}(x-a)]^{q}}=\frac{1}{\Gamma(-q)} \int_{a}^{x}(x-y)^{-q-1} f(y) \mathrm{d} y \quad(q<0),
$$

which can only be applied directly to fractional integration $(q<0)$, but can be extended to fractional differentiation by combining it with integer-order derivatives. It is beyond the scope of this paper to analyze these and other formulas of FC more thoroughly. Interested readers will find complete mathematical details in all the references cited in this section.

\section{Generalizing Newtonian Mechanics}

One-dimensional Newtonian mechanics for a point-particle of constant mass $m$ is based upon Newton's second law of motion, a second-order ordinary differential equation: 


$$
\frac{\mathrm{d}^{2} x(t)}{\mathrm{d} t^{2}}=\frac{F}{m} .
$$

We can easily think of at least two possible ways of generalizing Newton's second law using fractional calculus:

- Change the order of the time derivative in the left-hand-side of Equation (12) to an arbitrary number $q$. This is motivated by current studies of FC applied to physics [4], where second-order classical wave equations, Schrödinger and Dirac equations, and several others are generalized to fractional order $q$.

- Generalize the expression of the force $F$, or force field $F(x, t)$, on the right-hand-side of Equation (12) to include differintegrals of arbitrary order $q$. This is also routinely done in applications of FC to physics [4], by selecting fractional generalizations of standard electromagnetic potentials, in order to analyze phenomena in nuclear physics, hadron spectroscopy, and other fields.

In the next two sub-sections, we will consider examples of these possible generalizations.

\subsection{Constant Force Motion}

As our first example, we generalize Equation (12) by using derivatives of arbitrary (real) order $q$ and by considering a constant force per unit mass $f=F / m:^{2}$

$$
\frac{\mathrm{d}^{q} x(t)}{\mathrm{d} t^{q}}=\frac{F}{m}=f .
$$

The general solution of this (extraordinary) differential equation is [1]:

$$
\begin{aligned}
& x(t)=\frac{d^{-q} f}{d t^{-q}}+c_{1} t^{q-1}+c_{2} t^{q-2}+\cdots+c_{l} t^{q-l} \\
&=\frac{f t^{q}}{\Gamma(1+q)}+c_{1} t^{q-1}+c_{2} t^{q-2}+\cdots+c_{l} t^{q-l}, \\
& \text { with }\left\{\begin{array}{l}
0<q \leq l<q+1, \text { if } q>0 \\
l=0, \text { if } q \leq 0
\end{array}\right.
\end{aligned}
$$

having used also Equation (9) for the fractional derivative of the constant force per unit mass $f$. The $I$ constants of integration, $c_{1}, c_{2}, \cdots, c_{l}$, can be determined from the $l$ initial conditions: $x\left(t_{0}\right), x^{\prime}\left(t_{0}\right), \cdots, x^{(l-1)}\left(t_{0}\right) .^{3}$

For example, choosing for simplicity's sake $t_{0}=1,{ }^{4}$ the constants of integration are determined by a set of linear equations in matrix form $\boldsymbol{M c}=\boldsymbol{d}$, where

\footnotetext{
${ }^{2}$ We note that, in order to ensure the dimensional correctness of Equation (13), we would need to redefine force so that its dimensions become $M L T^{-q}$. Alternatively, if the customary dimensions of force are used, a constant time scale factor $t_{s c}$ should be introduced in Equation (13): $\frac{\mathrm{d}^{q} x(t)}{\mathrm{d} t^{q}}=\frac{F}{m} t_{S C}^{2-q}=f t_{S C}^{2-q}$. We have adopted the former solution in the following.

${ }^{3}$ Using non-integer derivatives in the initial conditions would greatly complicate the solution procedure, so we have avoided this unnecessary complication.

${ }^{4} \mathrm{We}$ prefer to avoid the customary choice of $t_{0}=0$, since the solutions described in Eq. $14 \mathrm{can}$ diverge for $t \rightarrow 0$.
} 
$\boldsymbol{c}=\left(c_{j}\right)$ is the vector of the integration constants, while the matrix $\boldsymbol{M}=\left(m_{i j}\right)$ and the vector $\boldsymbol{d}=\left(d_{i}\right)$ are obtained as follows:

$$
\begin{gathered}
m_{i j}=\left[\prod_{n=1}^{i-1}(q-j-n+1)\right]_{i, j=1,2, \cdots, l} \\
d_{i}=\left[x^{i-1}(1)-\frac{f}{\Gamma(1+q)} \prod_{n=0}^{i-2}(q-n)\right]_{i=1,2, \cdots, l}
\end{gathered}
$$

If the initial conditions and the force per unit mass are simply set to unity, i.e., $x(1)=x^{\prime}(1)=x^{\prime \prime}(1)=\cdots=1$ and $f=1$, our general solution in Equation (14), with the integration constants computed using Equations (15) and (16), can be easily plotted for different values of the order $q$, as shown in Figure 1.

This figure illustrates the resulting position vs. time functions for the point-particle motion, subject to the generalized Newton's law in Equation (13), with the order $q$ ranging from 1 to 3 with fractional increments. The standard Newtonian solution, $x(t)=\frac{1}{2} a\left(t-t_{0}\right)^{2}+v_{0}\left(t-t_{0}\right)+x_{0}$, is obviously recovered for $q=2$ (red-solid curve), for a motion with constant acceleration $a=F / m$. Two other solutions for integer values of $q$ are presented: the case for $q=1$ (blue-solid line) represents a simple motion with constant velocity $v=F / m$ : $x(t)=v\left(t-t_{0}\right)+x_{0}$; the $q=3$ case (green-solid line) represents instead a motion with constant jerk $k^{5} \quad j=F / m$ :

$$
x(t)=\frac{1}{6} j\left(t-t_{0}\right)^{3}+\frac{1}{2} a_{0}\left(t-t_{0}\right)^{2}+v_{0}\left(t-t_{0}\right)+x_{0}
$$

In Figure 1, we also show (dashed and dotted curves) the position vs. time functions for some fractional values of the order $q$ in Equation (13). These additional curves interpolate well between the integer-order functions described above, showing that "fractional mechanics" would simply yield solutions for the motion of the point-particle which are somewhat in-between the integer-order solutions.

One can't help but wonder what would the universe be like, if the fundamental Newtonian (second) law of motion were based on an order $q$ different from the standard value of two: for $q=1$, we would have a situation reminiscent of Aristotelian physics, where a constant applied force would only achieve a motion with constant velocity. For $q=3$, the application of a constant force would yield a constant jerk (i.e., an acceleration changing at constant rate) resulting in a motion much more difficult to control. Fractional values of $q$ would yield mechanical situations somewhat in-between those with integer $q$, but the resulting dynamics would possibly be lacking of the other cardinal principles of Newtonian mechanics, such as conservation laws or others.

\subsection{Gravitational Force}

Our second case of interest will be the generalization of Newton's law of

${ }^{5}$ The higher-order derivatives of the position vs. time function (beyond the second order) are usually called jerk (3rd order), snap or jounce (4th order), crackle (5th order), pop (6th order), etc. 


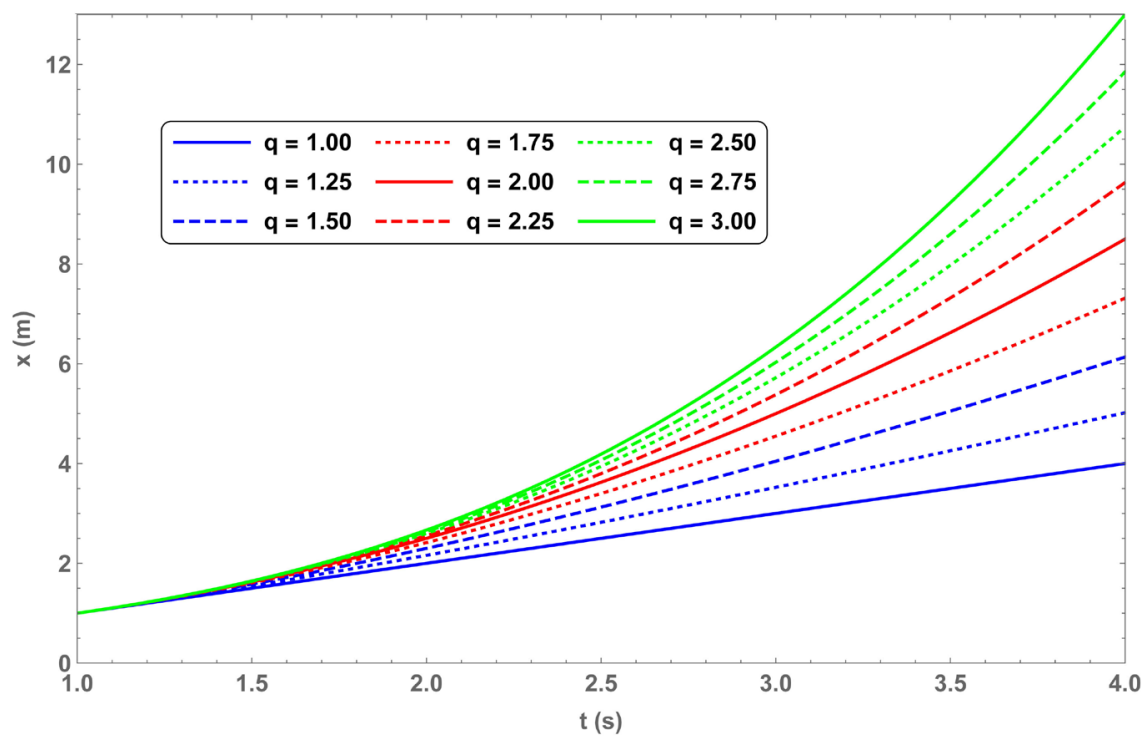

Figure 1. Position vs. time functions for a point-particle subject to a constant force, using the generalized Newton's law in Equation (13), with the order $q$ ranging from 1 to 3 , and with fractional increments. The standard Newtonian solution is recovered for $q=2$ (red-solid curve), for a motion with constant acceleration. The case for $q=1$ (blue-solid line) represents a motion with constant velocity, while the $q=3$ case (green-solid line) represents instead a motion with constant jerk. Other solutions (dashed and dotted curves), for some fractional values of the order $q$, are also shown in the figure.

universal gravitation:

$$
\frac{\mathrm{d}^{2} \boldsymbol{r}(t)}{\mathrm{d} t^{2}}=\frac{\boldsymbol{F}_{g}}{m}=-\frac{G M}{r^{2}} \hat{r},
$$

where $G$ is the universal gravitational constant, $M$ is the total mass of a (spherically symmetric) source centered at the origin of a coordinate system, $\hat{r}$ and $r$ are respectively the radial unit vector and the radial distance between the origin and a point-particle of mass $m$, subject to the gravitational attraction.

In this case, we will modify the right-hand-side of Equation (17) by considering a generalized gravitational Riesz potential [4] $V_{R Z}$ :

$$
V_{R Z}(\boldsymbol{r})=-\frac{G}{a} \int_{\mathbb{R}^{3}} \frac{\mathrm{d} M}{(s / a)^{q}}=-\frac{G}{a} \int_{\mathbb{R}^{3}} \frac{\rho\left(\boldsymbol{r}^{\prime}\right) \mathrm{d}^{3} \boldsymbol{r}^{\prime}}{\left(\left|\boldsymbol{r}-\boldsymbol{r}^{\prime}\right| / a\right)^{q}},
$$

where $s=\left|\boldsymbol{r}-\boldsymbol{r}^{\prime}\right|$ is the distance between the infinitesimal source mass element $\mathrm{d} M=\rho\left(\boldsymbol{r}^{\prime}\right) \mathrm{d}^{3} \boldsymbol{r}^{\prime}$ and the position $\boldsymbol{r}$ being considered. Due to the presence of the fractional order $q$, a "length scale" $a$ is needed to ensure the dimensional correctness of Equation (18). ${ }^{6}$

${ }^{6}$ This approach [4] is based on a 3D-generalization of the convolution integral:

$V(\boldsymbol{r})=\int_{\mathbb{R}^{3}} \rho\left(\boldsymbol{r}^{\prime}\right) w\left(\left|\boldsymbol{r}-\boldsymbol{r}^{\prime}\right|\right) \mathrm{d}^{3} \boldsymbol{r}^{\prime}=\int_{\mathbb{R}^{3}} \frac{\rho\left(\boldsymbol{r}^{\prime}\right)}{\left|\boldsymbol{r}-\boldsymbol{r}^{\prime}\right|} \mathrm{d}^{3} \boldsymbol{r}^{\prime}$, with a fractional weight function:

$w\left(\left|\boldsymbol{r}-\boldsymbol{r}^{\prime}\right|\right)=\frac{1}{\left|\boldsymbol{r}-\boldsymbol{r}^{\prime}\right|^{q}}$. The resulting potential in Equation (18) is equivalent to a 3D-version of the

Riesz fractional derivative, which corresponds to a linear combination of fractional Liouville integrals. 
For a spherical source of radius $R_{0}$ and uniform density $\rho_{0}=M /\left(\frac{4}{3} \pi R_{0}^{3}\right)$,

$$
\rho\left(\boldsymbol{r}^{\prime}\right)=\rho_{0} H\left(R_{0}-r^{\prime}\right)=\left\{\begin{array}{l}
\rho_{0}, \text { for } 0 \leq r^{\prime} \leq R_{0} \\
0, \text { for } r^{\prime}>R_{0}
\end{array}\right.
$$

the integral in Equation (18) can be evaluated analytically for any (real) value of the fractional order $q$, inside and outside the source. In general, we have [4]:

$$
\begin{aligned}
& V_{R Z}(r)=-\frac{G \rho_{0}}{r} \frac{2 \pi a^{q-1}}{(q-2)(q-3)(q-4)} \\
& \times\left\{\begin{array}{l}
{\left[\left(r+R_{0}\right)^{3-q}\left(r-(3-q) R_{0}\right)+\left(R_{0}-r\right)^{3-q}\left(r+(3-q) R_{0}\right)\right], \text { for } 0 \leq r \leq R_{0}} \\
{\left[\left(r+R_{0}\right)^{3-q}\left(r-(3-q) R_{0}\right)-\left(r-R_{0}\right)^{3-q}\left(r+(3-q) R_{0}\right)\right], \text { for } r>R_{0}}
\end{array}\right.
\end{aligned}
$$

for the inner and outer solutions. The special cases for $q=2,3,4$ can be obtained by recomputing the integrals for these particular values of $q$, or by considering appropriate limits of $V_{R Z}(r)$, from the previous equation, for $q \rightarrow 2,3,4$. For example, for $q=2$, we obtain:

$$
\left.V_{R Z}(r)\right|_{q=2}=-\frac{G \rho_{0}}{r} \pi a\left\{\begin{array}{l}
{\left[2 r R_{0}+\left(R_{0}^{2}-r^{2}\right) \ln \left(\frac{r+R_{0}}{R_{0}-r}\right)\right], \text { for } 0 \leq r \leq R_{0}} \\
{\left[2 r R_{0}-\left(r^{2}-R_{0}^{2}\right) \ln \left(\frac{r+R_{0}}{r-R_{0}}\right)\right], \text { for } r>R_{0} .}
\end{array}\right.
$$

Setting instead $q=1$ in Equation (20), and using $\rho_{0}=M /\left(\frac{4}{3} \pi R_{0}^{3}\right)$, we recover the standard Newtonian potential:

$$
\left.V_{R Z}(r)\right|_{q=1}=V_{\text {Newtonian }}(r)=\left\{\begin{array}{l}
-\frac{G M}{2 R_{0}^{3}}\left(3 R_{0}^{2}-r^{2}\right), \text { for } 0 \leq r \leq R_{0} \\
-\frac{G M}{r}, \text { for } r>R_{0},
\end{array}\right.
$$

which yields the universal law of gravitation in Equation (17), by using just the outer potential from the last equation. Another very simple case is the one for $q=0$, which yields a constant potential $\left.V_{R Z}(r)\right|_{q=0}=-\frac{G M}{a}$, for both inner and outer solutions.

In Figure 2, we illustrate the shape of these generalized gravitational Riesz potentials following Equations (20)-(22), for different values of the fractional order $q$ ranging from zero to two. The $q=1$ case (red-solid curve) represents the standard Newtonian gravitational potential. All these plots were obtained by setting $G=M=R_{0}=a=1$ for simplicity's sake, therefore the vertical grid line at $r=1.0$ in the figure denotes the boundary between the inner $\left(0 \leq r \leq R_{0}\right)$ and the outer $\left(r>R_{0}\right)$ potentials. As already mentioned above, the $q=0$ case (blue-solid line) corresponds to a constant potential $\left.V_{R Z}(r)\right|_{q=0}=-\frac{G M}{a}$, while the $q=2$ case (green-solid curve) is plotted using Equation (21).

An interesting consequence of these generalized gravitational potentials is the 


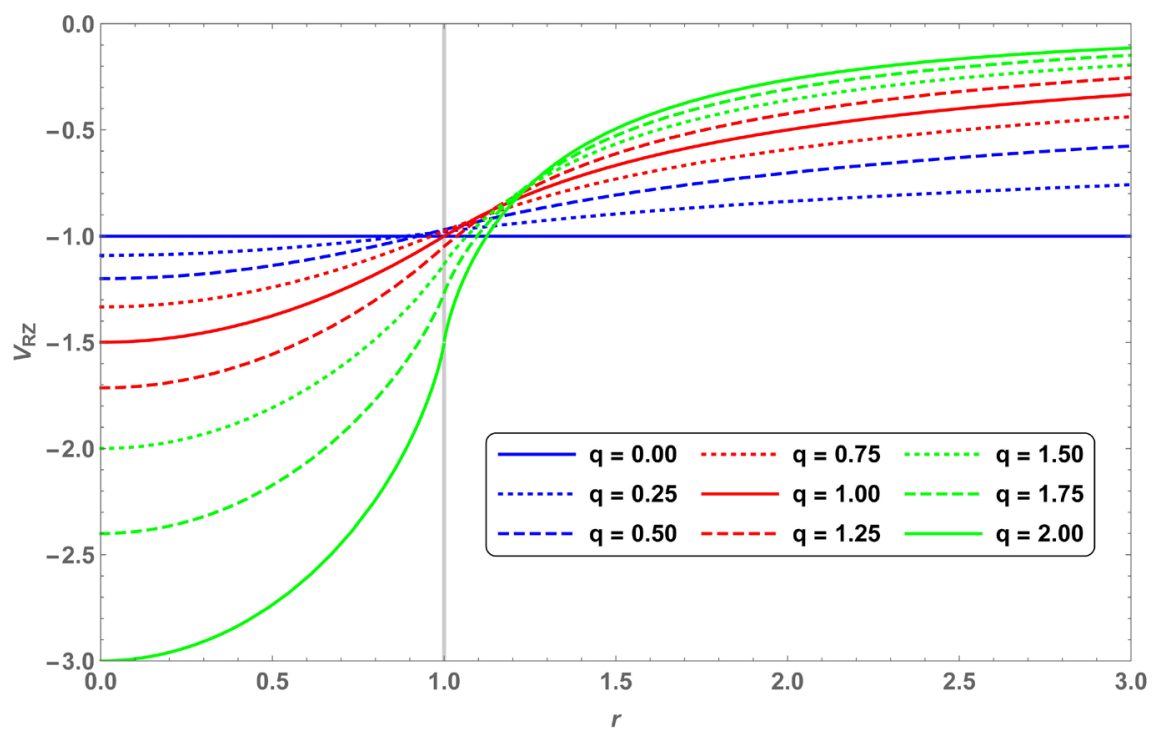

Figure 2. The generalized gravitational Riesz potentials, following Equations (20)-(22), for different values of the fractional order $q$. The $q=1$ case (red-solid curve) represents the standard Newtonian gravitational potential. We set $G=M=R_{0}=a=1$ for simplicity's sake, thus the vertical grid line at $r=1.0$ in the figure denotes the boundary between the inner $\left(0 \leq r \leq R_{0}\right)$ and the outer $\left(r>R_{0}\right)$ potentials. The $q=0$ case (blue-solid line) corresponds to a constant potential, while the $q=2$ case (green-solid curve) is plotted using Equation (21). Other potentials (dashed and dotted curves), for some fractional values of the order $q$, are also shown in the figure.

analysis of the resulting orbital circular velocities, for the inner and outer solutions. From the generalized gravitational potentials in Equation (20), we can easily obtain the related gravitational force per unit mass:

$$
\begin{aligned}
& \frac{\boldsymbol{F}_{R Z}(r)}{m}=-\frac{\mathrm{d} V_{R Z}(r)}{\mathrm{d} r} \hat{r}=-\frac{G \rho_{0}}{r^{2}} \hat{r} \frac{2 \pi a^{q-1}}{(q-2)(q-3)(q-4)} \\
& \times\left\{\begin{array}{l}
\left(r+R_{0}\right)^{2-q}\left(r-(3-q) R_{0}\right)\left(R_{0}-(2-q) r\right) \\
+\left(R_{0}-r\right)^{2-q}\left(r+(3-q) R_{0}\right)\left(R_{0}+(2-q) r\right) \\
-r\left(\left(r+R_{0}\right)^{3-q}+\left(R_{0}-r\right)^{3-q}\right), \text { for } 0 \leq r \leq R_{0} \\
\left(r+R_{0}\right)^{2-q}\left(r-(3-q) R_{0}\right)\left(R_{0}-(2-q) r\right) \\
+\left(r-R_{0}\right)^{2-q}\left(r+(3-q) R_{0}\right)\left(R_{0}+(2-q) r\right) \\
-r\left(\left(r+R_{0}\right)^{3-q}-\left(r-R_{0}\right)^{3-q}\right), \text { for } r>R_{0}
\end{array}\right.
\end{aligned}
$$

from which we can obtain the orbital circular velocities:

$$
v_{\text {circ }}(r)=\sqrt{r \frac{\left|\boldsymbol{F}_{R Z}(r)\right|}{m}}=\sqrt{r\left|\frac{\mathrm{d} V_{R Z}(r) \mid}{\mathrm{d} r}\right|} .
$$

Figure 3 shows the plots of these orbital circular velocities for the same values of the fractional order $q$ used in Figure 2, and also by setting $G=M=R_{0}=a=1$ as done previously. The $q=1$ case (red-solid curve) represents the standard 


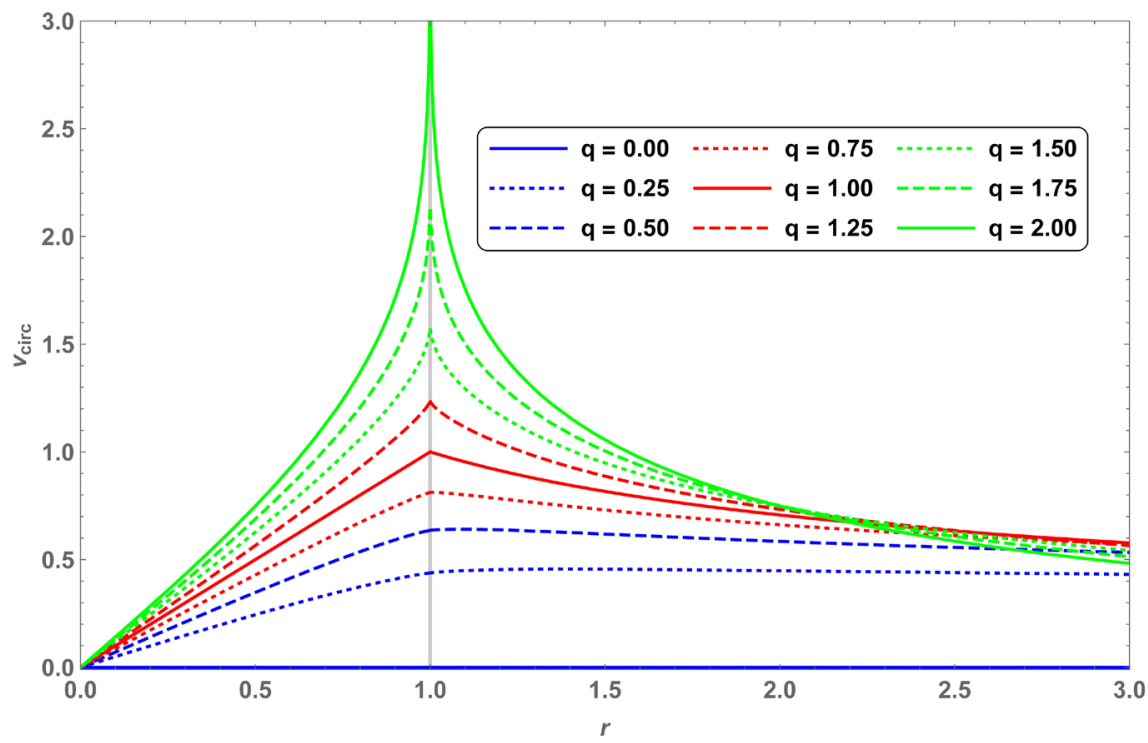

Figure 3. The orbital circular velocities, following Equations (23) and (24), for different values of the fractional order $q$. The $q=1$ case (red-solid curve) represents the standard Newtonian situation. Again, we set $G=M=R_{0}=a=1$, thus the vertical grid line at $r=1.0$ in the figure denotes the boundary between the inner $\left(0 \leq r \leq R_{0}\right)$ and the outer ( $r>R_{0}$ ) velocities. The $q=0$ case (blue-solid line) corresponds to a zero force situation, while the $q=2$ case (green-solid curve) is plotted using Equations (21) and (24). Other solutions (dashed and dotted curves), for some fractional values of the order $q$, are also shown in the figure.

Newtonian situation, with the circular velocity $v_{\text {circ }}=\sqrt{\frac{G M}{R_{0}^{3}}} r \sim r$ for $0 \leq r \leq R_{0}$, and $v_{\text {circ }}=\sqrt{\frac{G M}{r}} \sim 1 / \sqrt{r}$ for $r \geq R_{0}$ (the vertical grid line at $r=1.0$ in the figure represents the boundary between the inner and outer regions).

The $q=0$ case (blue-solid line) would not yield any circular velocity because it corresponds to a zero-force case. The $q=2$ case (green-solid curve) is computed using the special potential in Equation (21), while in all the other (fractional) cases the velocity plots interpolate well between the integer cases outlined above.

It is interesting to note that, for values of $q$ decreasing from one toward zero, the rotational velocity curves in the outer $\left(r \geq R_{0}\right)$ region show a definite "flattening" effect, which becomes more pronounced for the lowest $q$ values (for example, in the $q=0.25$ case, blue-dotted curve). This consideration might be of some interest in relation with the well-established problem of dark matter in galaxies, as evidenced by the galactic rotation curves and their lack of Newtonian behavior in the outer regions.

It is beyond the scope of this paper to perform any fitting of galactic rotational curves, by means of our fractional model of the Riesz gravitational potentials. However, it is interesting to note that the main feature of the observed galactic rotational curves, i.e., their conspicuous flatness at larger distances could be ac- 
tually recovered for values of the fractional order $q$ close to zero.

We also recall that one of the most popular alternative gravitational models, Modified Newtonian Dynamics (MOND) [6] [7], originated from a simple modification of Newton's second law, to account for the observed properties of galactic motion. The MOND modification can be applied to either side of Newton's second law [7]: by setting the force to be proportional to a certain function of the acceleration, or alternatively by changing the dependence of the gravitational force on the distance. In this work we have shown that similar modifications to the dynamics of a body in motion can also be obtained by means of fractional calculus. Also, given possible connections between fractional calculus and fractal geometry [8] [9] [10] [11] [12], a fractional approach to mechanics might be useful to analyze complex structures such as galaxies or similar.

\section{Conclusions}

In this work, we have applied fractional calculus to some elementary problems in standard Newtonian mechanics. The main goal was to show that FC can be used as a pedagogical tool, even in introductory physics courses, to gain more insight into basic concepts of physics, such as Newton's laws of motion and universal gravitation.

An intriguing consequence of FC, in connection with gravitational physics, is the possibility of applying fractional mechanics to the problem of galactic rotation curves. We will leave to further studies to investigate in more detail a possible connection between fractional mechanics and the dark matter puzzle.

\section{Acknowledgements}

This work was supported by a grant from the Frank R. Seaver College of Science and Engineering, Loyola Marymount University, Los Angeles. Sincere thanks to the members of JAMP for their professional performance, and special thanks to managing editor Jasmyn Chen for a rare attitude of high quality.

\section{References}

[1] Oldham, K.B. and Spanier, J. (1974) The Fractional Calculus. Academic Press, New York-London.

[2] Miller, K.S. and Ross, B. (1993) An Introduction to the Fractional Calculus and Fractional Differential Equations. Wiley-Interscience Publication. John Wiley \& Sons, Inc., New York.

[3] Podlubny, I. (1999) Fractional Differential Equations, Volume 198 of Mathematics in Science and Engineering. Academic Press, Inc., San Diego, CA.

[4] Herrmann, R. (2014) Fractional Calculus: An Introduction for Physicists. World Scientific Publishing Co., Inc., River Edge, NJ. https://www.worldscientific.com/worldscibooks/10.1142/8934

[5] Hilfer, R., Ed. (2000) Applications of Fractional Calculus in Physics. World Scientific Publishing Co., Inc., River Edge, NJ. https://doi.org/10.1142/3779

[6] Milgrom, M. (1983) A Modification of the Newtonian Dynamics as a Possible Al- 
ternative to the Hidden Mass Hypothesis. The Astrophysical Journal, 270, 365-370.

[7] Milgrom, M. (2001) MOND: A Pedagogical Review. Acta Physica Polonica B, 32, 3613.

[8] Tatom, F.B. (1995) The Relationship between Fractional Calculus and Fractals. Fractals, 3, 217-229. https://doi.org/10.1142/S0218348X95000175

[9] Nottale, L. (2010) Scale Relativity and Fractal Space-Time: Theory and Applications. Foundations of Science, 15, 101-152. https://doi.org/10.1007/s10699-010-9170-2

[10] Nottale, L. (2011) Scale Relativity and Fractal Space-Time. World Scientific Publishing Company, Singapore. https://doi.org/10.1142/p752

[11] Calcagni, G. (2017) Multiscale Spacetimes from First Principles. Physical Review D, 95, Article ID: 064057. https://doi.org/10.1103/PhysRevD.95.064057

[12] Calcagni, G. (2017) Multifractional Theories: An Unconventional Review. Journal of High Energy Physics, 2017, 138. https://doi.org/10.1007/JHEP03(2017)138 